\title{
Hirsutism in the United Arab Emirates: a hospital study
}

\author{
Omran B Gatee, Haider M Al Attia, Ismail A Salama
}

\begin{abstract}
Summary
In a study of 102 women (Arabs and Asians) with hirsutism, polycystic ovary syndrome was diagnosed in 93 patients (91\%) including 26 cases with hirsutism and regular menstrual periods. In more than half of the cases the diagnosis was made on the basis of transabdominal ultrasonography, which was the single test with the highest yield. Luteinising hormone hyperresponsiveness to gonadotropin-releasing hormone was positive in only seven of 50 patients tested. Late onset congenital adrenal hyperplasia due to 21-hydroxylase deficiency was identified in two cases, thus forming a prevalence of $8 \%$ in those who underwent adrenocorticotropin stimulation. In one case, polycystic ovary syndrome was an associated condition. In this study of a population of women with hirsutism and regular menses, the majority had polycystic ovaries. It is suggested that the term 'idiopathic or racial' hirsutism should not be applied unless a thorough evaluation has failed to reveal a diagnosable underlying disorder.
\end{abstract}

Keywords: hirsutism, polycystic ovary syndrome, Asians, Arabs

Hirsutism is a common clinical problem with different aetiologies which vary in frequency in different populations. ${ }^{1,2}$ The large range of familial and racial differences in the extent and acceptability of hair on various body sites makes objective assessment essential. However, various methods have been proposed to assess the degree of hirsutism. ${ }^{3-5}$ Careful endocrinological evaluation of women defined as having hirsutism have shown increased androgen metabolism in up to $90 \%{ }^{6}$ It has long been thought that racial or idiopathic hirsutism was the primary type found in the Middle Eastern community. ${ }^{7,8}$ This current study evaluated the most common androgenic aetiologies of hirsuitism in 102 consecutive patients presenting with hirsutism to an outpatient endocrinology service in Abu Dhabi.

Department of Internal Medicine Mafraq Hospital, Abu Dhabi, UAE

HM Al Attia

Correspondence to Dr OB Gatee, PO Box 2427, Al Jaziera Hospital, Abu Dhabi, UAE and two others cater to the medical needs of the Abu Dhabi population, and provide specialised medical services. The group includes UAE natives, non-UAE Arab expatriates and Asians (from the Indian subcontinent). The UAE community is multi-ethnic and these nationals reflect the bulk of the population.

All but 27 had a history of oligo/amenorrhoea since menarche. None had received any steroidal medications for at least eight weeks prior to being studied. Hirsutism was described as mild (Ferriman-Gallwey score 6-9), moderate $(10-14)$, or severe $(>15) .{ }^{3}$ Most of our patients fell between moderate to severe scores.

Eighty-seven patients (85\%) underwent pelvic ultrasonography, which was evaluable. In the rest, the examination was either inconclusive or declined by the patient. The ovary was defined as being polycystic, if there were multiple cysts (10 or more), $2-8 \mathrm{~mm}$ in diameter, arranged either peripherally around the stroma or distributed throughout an increased amount of stroma, or both. Enlargement of the ovaries (dimensions of normal adult female ovary based on anatomic specimen length $2.2-5.5 \mathrm{~cm}$, width $1.5-2 \mathrm{~cm}$, AP diameter of $1.5-3 \mathrm{~cm}$ ) was considered as a positive finding in this context. ${ }^{9,10}$ Morning samples for hormonal assessment, collected during the follicular phase (days 5-9) of the cycle included basal levels of the following hormones: luteinising hormone, follicle-stimulating hormone, prolactin, testosterone, dehydroepiandosterone, 17-hydroxyprogesterone, androstenedione and cortisol. Estimation of 24-hour free cortisol in urine was performed if Cushing's syndrome was clinically suspected. Fifty patients (49\%) had gonadotropin-releasing hormone stimulation test. The test was performed by administration of a single intravenous injection of $100 \mu \mathrm{g}$ gonadotropin-releasing hormone during the mid-follicular phase and blood samples were obtained before and 20 and 60 minutes after the injections. ${ }^{11}$

Twenty-five patients $(24.5 \%)$, some with raised basal levels of dehydroepiandosterone, testosterone and 17-hydroxyprogesterone (three to five times the basal level) and others who failed to respond to conventional treatment for polycystic ovaries (cyproterone acetate, spironolactone, clomiphene, contraceptive pills and bromocriptine for associated hyperprolactinaemia) underwent an adenreocorticotropin stimulation test. A single intravenous bolus of $0.25 \mathrm{mg}$ of synthetic adrenocorticotropin was given in the midfollicular phase at $08.00 \mathrm{~h}$; blood samples were 
obtained at 0,30 and 60 minutes for determination of serum 17-hydroxyprogesterone, progesterone, dehydroepiandosterone, cortisol and androstenodione. Assays of 11deoxycortisol, 17-hydroxypregnenolone and free testosterone were not available in our laboratories. Polycystic ovarian disease was diagnosed with a combination of clinical, biological and ultrasonographic parameters (box).

Diagnoses of Cushing's syndrome, acromegaly, prolactinoma, or thyroid disorders were excluded by performing the appropriate static and dynamic hormonal assays and in appropriate patients, radiological tests including computed tomography (CT).

\section{Results}

Of the 102 patients, 72 were Emirian nationals, 18 were Arab expatriates (non-UAE Arabs), and 12 were Asians from the Indian subcontinent. Their age ranged from 14-37 years with an average of 22.3 years. Sixty-three patients were obese (body mass index $>25 \mathrm{~kg} / \mathrm{m}^{2}$ ). In the 50 patients who underwent a gonadatropinreleasing hormone stimulation test, an enhanced response as defined by Marshall $e t$ $a l^{11}$ (ie, at least a four-fold rise of the basal level of luteinising hormone at 20 minutes after the gonadotropin-releasing hormone injection) was observed in only seven patients $(14 \%)$. Of these, only one had abnormal ultrasonographic findings consistent with polycystic ovary syndrome. An enhanced response of 17hydroxyprogesterone (10 times or more the basal level) to adrenocorticotropin stimulation was obtained in only two cases of the 25 patients tested. These two patients were thus diagnosed as late-onset congenital adrenal hyperplasia due to 21-hydroxylase deficiency. In the one patient in whom 17-hydroxyprogesterone suppression was attempted, the basal level was suppressible by dexamethasone; this patient also had ultrasonographic features consistent with polycystic ovary syndrome. In the whole group, 93 patients $(91 \%)$ were diagnosed as having polycystic ovary syndrome alone. These included 26 patients who had previously been considered to have idiopathic hirsutism (hirsutism with regular menstrual periods). In this group, hormonal changes consistent with polycystic ovary syndrome (high basal level of serum luteinising hormone, testosterone, androstenedione, and high luteinising hormone/ follicle-stimulating hormone ratio) were observed in $20 / 26(77 \%)$ and positive ultrasonographic changes of polycystic ovary syn-

\begin{tabular}{|l|}
\hline $\begin{array}{l}\text { Diagnostic criteria for polycystic } \\
\text { ovaries }\end{array}$ \\
\hline Two of the following groups occurring together: \\
- amenorrhoea, oligomenorrhoea, \\
non-mechanical sterility or hirsutism \\
biological hyperandrogenism (increased \\
testosterone and/or androstenedione) \\
- ultrasonographic abnormalities 9,10 \\
\hline
\end{tabular}

drome in $12 / 23(52 \%)$. The relevant data are shown in table 1. An ovarian tumour was diagnosed in one patient who presented with hirsutism, virilisation and recent menstrual changes. She showed a very high basal level of serum testosterone along with positive radiological findings including $\mathrm{CT}$ scan. Another patient was proven to have bilateral dermoid cyst by laparotomy and histopathology. Five remaining patients were classified as having 'uncategorised hirsutism' on the basis of upper limit values of serum testosterone alone. The rest of their hormonal assays and ultrasonographic findings were within normal limits. These patients underwent adrenocorticotropin stimulation test to rule out partial or minor forms of late onset congenital adrenal hyperplasia. Their responses were also within normal limits. The aetiology of the diagnosis of hirsutism is shown in table 2.

\section{Discussion}

Both ovaries and adrenals are the sources of androgens. A classic cause of hirsutism is polycystic ovary disease; this was first recognised as the clinical triad of amenorrhoea, hirsutism and obesity by Stein and Leventhal some 60 years ago. ${ }^{12}$ Many women who complain of excess hair, however, are not obese, nor do they have menstrual disturbances. These women are often labelled as having idiopathic hirsutism. This diagnosis, however, was seriously challenged by Adams et al after reporting a high prevalence of polycystic ovary syndrome $(92 \%)$ in a sample of women with hirsutism and normal menses. ${ }^{10}$

Table 1 Laboratory findings of 93 patients with polycystic ovary syndrome alone. Normal ranges in parentheses

\begin{tabular}{|c|c|c|}
\hline Test & $n$ & $(\%)$ \\
\hline Positive ultrasound & $50 / 87$ & $(57)$ \\
\hline $\begin{array}{l}\text { Raised serum concentration of } \\
\text { luteinising hormone }(0.7-8.0 \mathrm{mIU} / \mathrm{ml})\end{array}$ & $44 / 93$ & (47) \\
\hline $\begin{array}{l}\text { Raised luteinising hormone/ } \\
\text { follicle-stimulating hormone } \\
\text { ratio }(>2)\end{array}$ & $40 / 93$ & (43) \\
\hline $\begin{array}{l}\text { High serum concentration of testo- } \\
\text { sterone }(0.06-0.8 \mathrm{ng} / \mathrm{ml})\end{array}$ & $60 / 93$ & (65) \\
\hline $\begin{array}{l}\text { High serum concentration of } \\
\text { androstenedione }(0.2-3.1 \mathrm{ng} / \mathrm{ml})\end{array}$ & $29 / 69$ & (42) \\
\hline $\begin{array}{l}\text { High serum concentration of dehydro- } \\
\text { epiandosterone }(350-4300 \mathrm{ng} / \mathrm{ml})\end{array}$ & $15 / 77$ & (19.5) \\
\hline
\end{tabular}

Table 2 Aetiology of the diagnosis of hirsutism in the whole group

\begin{tabular}{lrl}
\hline Diagnosis & $n$ & $(\%)$ \\
\hline Polycystic ovary syndrome (alone) & 93 & 91 \\
Polycystic ovary syndrome and & 1 & $(1)$ \\
$\quad$ congenital adrenal hyperplasia & & \\
Congenital adrenal hyperplasia (alone) & 1 & $(47)$ \\
Ovarian tumour & 1 & \\
Bilateral dermoid cyst & 1 & $(1)$ \\
Uncategorised & 5 & $(5)$ \\
\hline
\end{tabular}


In our group the relatively high yield of positive pelvic ultrasonography in the diagnosis of polycystic ovary syndrome appeared to make it the investigation of choice in the evaluation of hirsutism. The result is comparable to that reported in a series of 350 patients in the UK $(60 \%){ }^{2}$ Even higher results were obtained by Adams et al among hirsutes with menstrual disturbances $(87 \%)$ and in others with regular periods $(92 \%){ }^{10}$ These rates are operator-dependent and possibly reflect the more accurate assessment provided by the use of a vaginal transducer which usually allows closer access to the ovaries. ${ }^{13}$ Such a procedure was not feasible in our patients for socio-legal reasons.

Variations in basal levels of luteinising hormone in polycystic ovary syndrome are not unusual. Franks reported that $50-70 \%$ of patients with polycystic ovary syndrome exhibit a high basal level of luteinising hormone. ${ }^{14}$ Adams $^{10}$ and Grulet ${ }^{15}$ observed the same finding at rates of $66 \%$ and $62 \%$, respectively. The proportion of patients with a raised luteinising hormone/follicle-stimulating hormone ratio in our series was lower than those reported by Adams et $a l^{10}(66 \%)$ in the UK and by Yilmaz et al in their series of 31 Turkish women $(55 \%) .{ }^{16}$ The value of dynamic endocrine investigation in polycystic ovary syndrome has been extensively emphasized and luteinising hormone hyperresponsiveness to the gonadotropin-releasing hormone stimulation test has been advocated as the mainstay of diagnosis. ${ }^{15,17,18}$ Since few patients in our series showed an enhanced response of luteinising hormone to gonadotropin-releasing hormone stimulation, the stimulation test was of less value than ultrasonography in the diagnosis of polycystic ovary syndrome in our patients.

The frequency of polycystic ovary syndrome in different populations is variable. It appeared extremely high in our group (91\%), compared to the $62 \%$ and $60 \%$ found in the studies of Adams $^{10}$ and O'Driscoll, ${ }^{2}$ respectively. This study indicates that an initial clinical diagnosis of idiopathic hirsutism in a Middle Eastern population may more often be found to be polycystic ovary syndrome after investigation. This is in agreement with the views of Adams and colleagues who suggested that the idiopathic label was inappropriate for most patients with hirsutism and regular periods. ${ }^{10}$

While polycystic ovary syndrome remains

1 Eldar-Geva T, Hurwitz A, Vecsei P, et al. Secondary biosynthetic defects in women with late-onset congenital adrenal hyperplasia. $N$ Engl f Med 1990; 13: 855-63.

2 O'Driscoll JB, Mautora H, Higginson J, Pollack A, Kane J, Anderson DC. A prospective study of the prevalence of Anderson DC. A prospective study of the prevalence of
clear-cut endocrine disorders and polycystic ovaries in 350 patients presenting with hirsutism or androgenic alopecia. patients presenting with hirsutism

3 Ferriman D, Gallwey JD. Clinical assessment of body hair growth in women. $f$ Clin Endocrinol Metab 1961; 21: 440-7.

4 Hatch R, Rosenfield RL, Kim MH, Tredway D. Hirsutism: implications, etiology, and management. Am $\mathcal{f}$ Obste Gynecol 1981; 140: 815-30.

5 Cooke ID, Sawers RS. Investigation of hirsutism and selection of patients for treatment. In: Jeffcoate SL, ed. Androgens and anti-androgen therapy. New York: John Wiley \& Sons, 1982; p 95.

6 Abraham GE, Chakmakjian ZH. Plasma steroids in hirsutism. Obstet Gynecol 1974; 44: 171-5. the commonest cause of hyperandrogenemia and hirsutism, other more morbid causes need to be ruled out. Late onset congenital adrenal hyperplasia due to 21-hydroxylase deficiency appears to have variable ethnic distribution. Elder-Geva et al reported a prevalence of $8 \%$ of this enzymatic defect in 170 Israeli Jewish women with hirsutism, ${ }^{1}$ whereas this defect has been found in $2-6 \%$ of patients with oligomenorrhoea or hirsutism in the UK. ${ }^{19,20}$ Others have estimated frequencies ranging from $1-30 \%{ }^{1}$ Obviously, in our series, the number of late-onset congenital adrenal hyperplasia cases due to 21-hydroxylase deficiency is comparatively small, and neither of the two patients with this defect is of Arab origin. The blunted response of dehydroepiandosterone to adrenocorticotropin stimulation in our series may argue against the diagnosis of $3 \beta$-hydroxylase deficiency in these patients. Nevertheless, when facilities become available to us, the screening for appropriate steroid intermediates (17-hydroxypregnenolone, and 11-deoxycortisol for 11-hydroxylase deficiency) will be imperative. The presence of co-existing polycystic ovary syndrome in one patient with late-onset congenital adrenal hyperplasia emphasizes that this ovarian finding does not rule out the presence of other important pathology.

Some authors advocate the use of the adrenocorticotropin stimulation test in every patient with hirsutism, ${ }^{1}$ but we tend to believe that their opinion may be influenced by the fact that late-onset congenital adrenal hyperplasia is frequently encountered in their community. The test is not cost-effective and the majority of our patients have radiological and hormonal changes in keeping with polycystic ovary syndrome, thus performing the adrenocorticotropin stimulation test in hirsutism routinely is probably of limited practical value in this community. The test is better reserved for those patients who do not respond to the conventional treatment for polycystic ovary syndrome. Finally, there is a need for further studies to evaluate hirsuitism in each major ethnic group of this community. The magnitude of 'racial hirsutism' remains obscure. Large number of families will need to be evaluated in order to determine its prevalence.

We thank Dr M Al Bitar and Mrs Mariam Mathew for their assistance in this study.

7 Baker C. McFarland KF. Hirsutism. In: Rakel RE, ed. Text book of family practice. Philadelphia: WB Saunders, 1990; p 1197.

p 1197.

8 Bates GW. Hirsutism and androgen excess in childhood and adolescence. Pediatr Clin N Am 1981; 28: 513-30.

9 Swanson M, Sauerbrei EE, Cooperberg PL. Medical implications of ultrasonically detected polycystic ovaries. $\mathcal{f}$ Clin Ultrasound 1981; 9: 219-22.

10 Adams J, Polson DW, Frank S. Prevalence of polycystic ovaries in women with anovulation and idiopathic hirsutism. $B M F$ 1986; 293: 355-9.

1 Marshall JC, Besser GM. Applications of the LH/FSHreleasing hormone. Joint meeting of the section of Endocrinology with the Society for Endocrinology Royal Society of Medicine, 1973.

2 Stein IF, Leventhal MC. Amenorrhoea associated with bilateral polycystic ovaries. Am 7 Obstet Gynecol 1935; 29: $181-91$. 
13 Fox R, Corrigan E, Thomas PA, Hull MGR. The diagnosis of polycystic ovaries in women with oligoamenorrhoea: predictive power of endocrine tests. Clin Endocrinol 1991; 34: $127-31$.

14 Franks S. Polycystic ovary syndrome: a changing perspective. Clin Endocrinol 1989; 31: 87-120.

15 Grulet H, Hecart AC, Delemer B, et al. Roles of LH and insulin resistance in lean and obese polycystic ovary syndrome. Clin Endocrinol 1993; 38: 621-6.

16 Yilmaz S, Fahrettin K. 17-Hydroxyprogesterone response to buserlin testing in the polycystic ovary syndrome. Clin Endocrinol 1993; 39: 151-5.

17 Barnes RB, Rosenfield RL. The polycystic ovary syndrome pathogenesis and treatment. Ann Intern Med 1989; 110 386-99.
18 McKenna TJ. Pathogenesis and treatment of polycystic ovary syndrome. $N$ Engl $₹$ Med 1988; 318: 558-62.

19 Hague WM, Honour JW, Adams J, Vecsei P, Jacobs HS. Steroid response to ACTH in women with polycystic ovaries. Clin Endocrinol 1989; 30: 355-65.

20 Turner EL, Watson MJ, Perry LA, White MC. Investigation of adrenal function in women with oligomenorrhoea and hirsutism (clinical PCOS) from the north-east of England using adrenal stimulation test. Clin Endocrinol 1992; 36: 389-97. 